\title{
Avaliação da proficiência em mapeamento conceitual a partir da análise estrutural da rede proposicional
}

\author{
Proficiency assessment in concept mapping \\ from the structural analysis of the propositional network
}

Paulo Rogério Miranda Correia ${ }^{1}$. Joana Guilares de Aguiar ${ }^{2}$

\begin{abstract}
Resumo: Os mapas conceituais (MCs) são organizadores gráficos que permitem representar o conhecimento e promover a aprendizagem significativa. A utilização adequada da técnica em sala de aula depende do treinamento dos alunos. Este trabalho teve como objetivo avaliar e acompanhar a proficiência dos alunos a partir da análise da estrutura da rede proposicional dos MCs. Para isso foi proposto o uso da Análise Estrutural de $434 \mathrm{MCs}$ produzidos pelos alunos durante a disciplina Ciências da Natureza na Escola de Artes, Ciências e Humanidades (EACH/USP) nas 1 1a $5^{\mathrm{a}}$ e $15^{\mathrm{a}}$ aulas. A partir de análises estatísticas univariada e multivariada dos dados foi possível inferir que a densidade proposicional e a quantidade de conceitos múltiplos iniciais e finais são parâmetros suficientes para determinar a proficiência dos alunos na técnica de mapeamento, a qual foi estabelecida à medida que as tarefas de treinamento e elaboração dos MCs foram acontecendo durante as 15 aulas da disciplina.
\end{abstract}

Palavras-chave: Mapa conceitual. Análise estrutural. Ensino superior. Ensino de ciências. Aprendizagem significativa.

\begin{abstract}
Concept maps (Cmaps) are graphic organizers that represent knowledge and foster meaningful learning. The proper use of the technique in the classroom depends on training students. This study aimed to assess and track students' proficiency from the structural analysis of the Cmap propositional network. We proposed the use of Structural Analysis of 434 Cmaps collected during the Natural Science Course (NSC) at the School of Arts, Sciences and Humanities during $1^{\text {st }}, 5^{\text {th }}$ and $15^{\text {th }}$ classes. Statistical univariate and multivariate data analysis let us infer that the propositional density and the quantity of initial and final multiple concepts are both useful parameters to assess the students' proficiency in the mapping technique, which was established throughout training, and Cmap elaboration tasks during the fifteen classes.
\end{abstract}

Keywords: Concept maps. Structural analysis. Higher education. Science education. Meaningful learning.

\footnotetext{
${ }^{1}$ Universidade de São Paulo (USP), Escola de Artes, Ciências e Humanidades, São Paulo, SP, Brasil. E-mail <prmc@usp.br>.

${ }^{2}$ Universidade de São Paulo (USP), Programa de Pós-graduação Interunidades em Ensino de Ciências, São Paulo, SP, Brasil.
} 


\section{Introdução}

Na década de 1970, Joseph Novak e colaboradores propuseram a utilização dos Mapas Conceituais (MCs) para representar as mudanças conceituais dos alunos sobre temas científicos ao longo da escolarização básica (NOVAK; MUSONDA, 1991). Em 1990, o reconhecimento da aplicação dos MCs no Ensino de Ciências se confirmou com a publicação da edição especial sobre o tema no periódico Journal of Research in Science Teaching (p. ex., NOVAK, 1990; STARR; KRAJCIK, 1990; WANDERSEE, 1990). Apesar do interesse crescente da comunidade científica pelos MCs e a disseminação da sua utilização em outras áreas do conhecimento (p. ex., CORREIA et al., 2016; MOON et al., 2011; NOVAK, 2010; TORRES; MARRIOT'T, 2010), as aplicações mais numerosas ainda se relacionam com o Ensino de Ciências.

O mapeamento conceitual possui uma forte vinculação com a Teoria da Assimilação através da Aprendizagem e da Retenção Significativas, proposta por David Ausubel (AUSUBEL, 2000; MOREIRA, 2011). As proposições, formadas por conceito inicial - termo de ligação $\rightarrow$ conceito final, diferenciam os MCs de outros diagramas similares, tais como os mapas mentais e os mapas de argumentos (DAVIES, 2011). As proposições obrigam a externalização das relações conceituais, que devem ser explicadas da forma mais clara possível através do uso de termos de ligação contendo verbo.

Os MCs revelam explicitamente a relação entre os conceitos (aspecto semântico), ao mesmo tempo em que organizam a informação de forma diagramática (aspecto visuoespacial). Isso potencializa o processamento do conteúdo pela memória de trabalho, visto que ela é capaz de lidar simultaneamente com estímulos verbais e imagéticos (PAIVIO, 1990). Considerando a escassez de recursos cognitivos da memória de trabalho, o uso dos MCs auxilia a aprendizagem ativa e, consequentemente, a aprendizagem significativa (NOVAK, 2010; SWELLER; AYRES; KALYUGA, 2011; VEKIRI, 2002). Nesse contexto, o mapeamento conceitual é repetidamente descrito na literatura como potencial ferramenta de ensino para a promoção da aprendizagem dos alunos sobre conceitos científicos (p. ex., FISCHER et al., 2002; HAY; KINCHIN; LYGO-BAKER, 2008; NESBIT; ADESOPE, 2006; NOVAK, 1990; RUIZ-PRIMO; SHAVELSON, 1996).

Apesar de ser uma técnica bem estabelecida e amplamente utilizada no âmbito educacional, alguns obstáculos ainda precisam ser superados para que os MCs sejam adotados de forma mais frequente pelos professores de Ciências. A aparente facilidade na elaboração de MCs explica sua popularidade, ainda que o seu uso ingênuo produza poucos (ou nenhum!) dos benefícios esperados (CORREIA; INFANTE-MALACHIAS; GODOY, 2008). Alguns trabalhos na literatura mostram que muitas das dificuldades encontradas para a plena adoção dos MCs derivam, pelo menos em parte, do uso inadequado da técnica, do treinamento ineficaz ou inexistente de alunos e professores, e da pouca importância dada aos fundamentos teóricos subjacentes ao mapeamento conceitual como, por exemplo, o entendimento sobre as proposições como unidade semântica, a organização hierárquica dos conceitos e a assimilação por meio da aprendizagem significativa (AGUIAR; CORREIA, 2013; CAÑAS; NOVAK, 2006; CONRADTY; BOGNER, 2010; HILBERT; RENKL, 2008; NOVAK, 2010).

Um período de treinamento na técnica pode evitar o uso ingênuo dos MCs em sala de aula. Nesses casos, verifica-se o estímulo à aprendizagem significativa e aos processos co- 
laborativos como principais dividendos capazes de mudar a dinâmica social em sala de aula. Trabalhos recentes confirmam que há espaço na literatura para pesquisas mais aprofundadas sobre os efeitos de treinamento e estratégias de capacitação de usuários iniciantes, assim como o desenvolvimento de critérios de avaliação desse processo. Por exemplo, Karpicke e Blunt (2011) destacam essa necessidade de pesquisa quando mencionam em artigo publicado na Science que:

Não encontramos nenhum estudo que manipula o treinamento para analisar se o mesmo melhora a eficiência do mapeamento conceitual. Dada a importância de identificar as melhores formas de implementar estratégias eficazes, é surpreendente que o papel do treinamento no mapeamento conceitual não esteja sendo rigorosamente examinado (KARPICKE; BLUNT, 2011, p. 453-d, tradução nossa).

\section{A análise estrutural de mapas conceituais e a relação com a proficiência do mapeador}

Há, na literatura, trabalhos importantes que descrevem formas de analisar a morfologia da rede proposicional dos MCs (p. ex., GERSTNER; BOGNER, 2009; KINCHIN; ALIAS, 2005). Dentre eles, Kinchin, Hay e Adams (2000) propõem uma abordagem qualitativa para a análise estrutural dos MCs. Os três padrões identificados pelos autores (radial, cadeia e rede) guardam estreita relação com a compreensão do aluno sobre o conteúdo do MC - quanto maior o grau de interconexões entre as proposições, maior o conhecimento do aluno sobre o conteúdo mapeado. Cañas et al. (2006) propõem uma taxonomia topológica que classifica os MCs em seis possíveis categorias em termos de suas características estruturais e semânticas. Nesse caso, tais categorias indicam um aumento progressivo na complexidade e qualidade dos MCs, que pode ser obtido a partir do domínio gradual da técnica e do conteúdo em estudo.

Dois trabalhos publicados nas atas das Conferências Internacionais sobre Mapeamento Conceitual também merecem destaque. O primeiro apresenta a aplicação do programa computacional CmapAnalysis, que permite detalhada análise quantitativa, automatizada e personalizada de diversos parâmetros estruturais dos MCs (CAÑAS; BUNCH; REISKA, 2010). Apesar de ser uma proposta promissora, o programa não é de simples manipulação e os dados de saída não são interpretados facilmente. O segundo artigo faz extensa discussão sobre o efeito de se manipular a liberdade/restrição de estrutura e conteúdo para a elaboração e, consequentemente, qualidade dos MCs (CAÑAS; NOVAK; REISKA, 2015).

Os trabalhos discutidos acima nos inspiraram a desenvolver uma Análise Estrutural (AE) quantitativa para capturar características morfológicas da rede proposicional dos MCs (SILVA JR.; ROMANO JR.; CORREIA, 2010). A AE contém oito parâmetros facilmente contabilizados a partir da quantidade de proposições e conceitos. A Figura 1 traz um MC genérico para exemplificar a AE e a Tabela 1 explica a análise em detalhes. Observe que todos os parâmetros são calculados em função da razão da quantidade total de conceitos do $\mathrm{MC}\left(\mathrm{C}_{\mathrm{T}}\right)$. Isso garante parâmetros com valores relativos que independem do tamanho do MC elaborado. 
Figura 1. Representação esquemática de um mapa conceitual contendo 6 conceitos (C1-C6) e 6 proposições
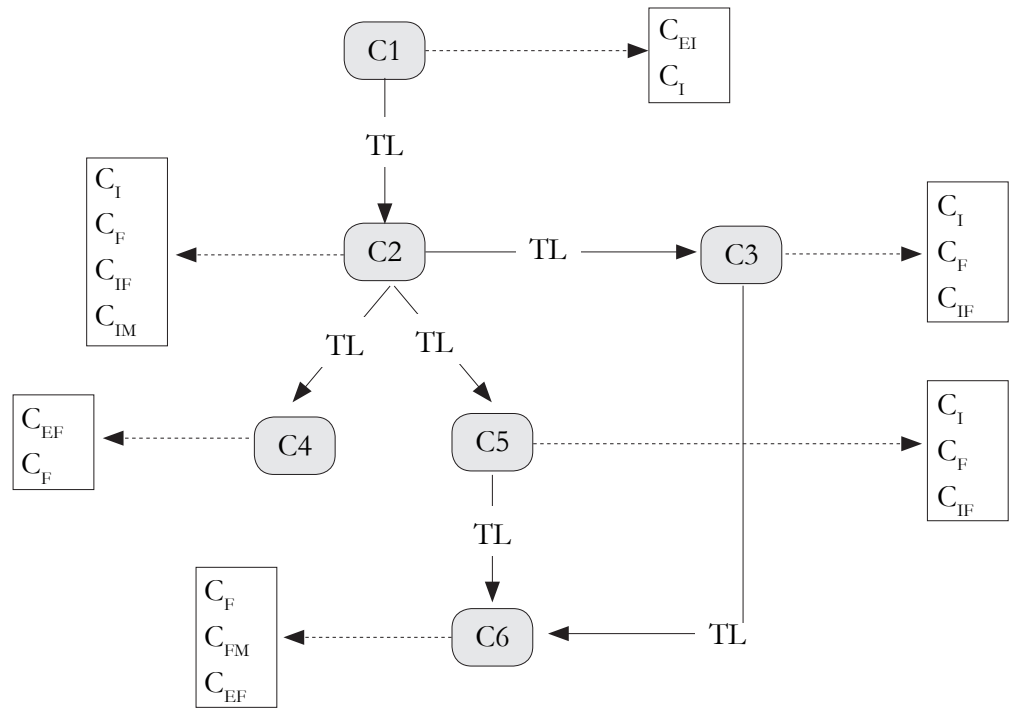

A classificação dos conceitos segundo a Análise Estrutural está indicada nos retângulos. Acrônimos e exemplos são descritos na Tabela 1. TL indica termo de ligação.

Fonte: elaborada pelos autores.

Tabela 1. Descrição dos parâmetros da Análise Estrutural dos mapas conceituais

\begin{tabular}{|c|c|c|c|}
\hline Parâmetro & Simbologia & Descrição & Exemplo \\
\hline Densidade Proposicional & $\mathrm{DP}=\mathrm{P} / \mathrm{C}_{\mathrm{T}}$ & $\begin{array}{l}\text { Razão entre a quantidade de } \\
\text { proposições }(\mathrm{P}) \text { e a quantidade } \\
\text { total de conceitos }\left(\mathrm{C}_{\mathrm{T}}\right) \text { no MC. }\end{array}$ & $\frac{P}{C_{T}}=\frac{6}{6}=1,00$ \\
\hline Conceitos Iniciais & $\mathrm{CI}=\mathrm{C}_{\mathrm{I}} / \mathrm{C}_{\mathrm{T}}$ & $\begin{array}{l}\mathrm{C}_{\mathrm{I}} \text { : apresentam uma ou mais } \\
\text { setas saindo da caixa do conceito, } \\
\text { podendo apresentar seta }(\mathrm{s}) \\
\text { chegando à caixa do conceito. }\end{array}$ & $\frac{\mathrm{C}_{\mathrm{I}}}{\mathrm{C}_{\mathrm{T}}}=\frac{4}{6}=0,67$ \\
\hline $\begin{array}{l}\text { Conceitos Iniciais } \\
\text { Múltiplos }\end{array}$ & $\mathrm{CIM}=\mathrm{C}_{\mathrm{IM}} / \mathrm{C}_{\mathrm{T}}$ & $\begin{array}{l}\mathrm{C}_{\mathrm{IM}} \text { : apresentam mais de } \\
\text { uma seta saindo da caixa do } \\
\text { conceito, podendo apresentar } \\
\text { seta(s) chegando à caixa do } \\
\text { conceito. }\end{array}$ & $\frac{\mathrm{C}_{\mathrm{IM}}}{\mathrm{C}_{\mathrm{T}}}=\frac{1}{6}=0,17$ \\
\hline
\end{tabular}


Tabela 1. continuação

\begin{tabular}{|c|c|c|c|}
\hline Parâmetro & Simbologia & Descrição & Exemplo \\
\hline Conceitos Finais & $\mathrm{CF}=\mathrm{C}_{\mathrm{F}} / \mathrm{C}_{\mathrm{T}}$ & $\begin{array}{l}\mathrm{C}_{\mathrm{F}} \text { : apresentam uma ou mais setas } \\
\text { chegando à caixa do conceito, } \\
\text { podendo apresentar seta(s) saindo } \\
\text { da caixa do conceito. }\end{array}$ & $\frac{\mathrm{C}_{\mathrm{F}}}{\mathrm{C}_{\mathrm{T}}}=\frac{5}{6}=0,83$ \\
\hline Conceitos Finais Múltiplos & $\mathrm{CFM}=\mathrm{C}_{\mathrm{FM}} / \mathrm{C}_{\mathrm{T}}$ & $\begin{array}{l}\mathrm{C}_{\mathrm{FM}}: \text { apresentam mais de uma } \\
\text { seta chegando à caixa do } \\
\text { conceito, podendo apresentar } \\
\text { seta }(\mathrm{s}) \text { saindo da caixa do } \\
\text { conceito. }\end{array}$ & $\frac{\mathrm{C}_{\mathrm{FM}}}{\mathrm{C}_{\mathrm{T}}}=\frac{1}{6}=0,17$ \\
\hline Conceitos Iniciais e Finais & $\mathrm{CIF}=\mathrm{C}_{\mathrm{IF}} / \mathrm{C}_{\mathrm{T}}$ & $\begin{array}{l}\mathrm{C}_{\mathrm{IF}} \text { : apresentam uma ou mais } \\
\text { seta }(\mathrm{s}) \text { saindo e uma ou mais } \\
\text { seta }(\mathrm{s}) \text { chegando à caixa do } \\
\text { conceito. }\end{array}$ & $\frac{\mathrm{C}_{\mathrm{IF}}}{\mathrm{C}_{\mathrm{T}}}=\frac{3}{6}=0,50$ \\
\hline $\begin{array}{l}\text { Conceitos Exclusivamente } \\
\text { Iniciais }\end{array}$ & $\mathrm{CEI}=\mathrm{C}_{\mathrm{EI}} / \mathrm{C}_{\mathrm{T}}$ & $\begin{array}{l}\mathrm{C}_{\mathrm{EI}} \text { : apresentam somente uma ou } \\
\text { mais seta }(\mathrm{s}) \text { saindo da caixa do } \\
\text { conceito, sem apresentar seta }(\mathrm{s}) \\
\text { chegando à caixa do conceito. }\end{array}$ & $\frac{\mathrm{C}_{\mathrm{EI}}}{\mathrm{C}_{\mathrm{T}}}=\frac{1}{6}=0,17$ \\
\hline $\begin{array}{l}\text { Conceitos Exclusivamente } \\
\text { Finais }\end{array}$ & $\mathrm{CEF}=\mathrm{C}_{\mathrm{EF}} / \mathrm{C}_{\mathrm{T}}$ & $\begin{array}{l}\mathrm{C}_{\mathrm{EF}} \text { : apresentam uma ou } \\
\text { mais seta(s) chegando à caixa } \\
\text { do conceito, sem apresentar } \\
\text { seta(s) saindo da caixa do } \\
\text { conceito. }\end{array}$ & $\frac{C_{\mathrm{EF}}}{\mathrm{C}_{\mathrm{T}}}=\frac{2}{6}=0,34$ \\
\hline
\end{tabular}

Os valores dados como exemplos consideraram o mapa conceitual apresentado na Figura 1. Acrônimos para os valores absolutos estão com o subscrito (p. ex. $C_{\text {IM }}$ ). Acrônimos para os valores relativos, calculados em função do número de conceitos totais $\left(\mathrm{C}_{\mathrm{T}}\right)$, estão sem subscrito (p. ex. CIM).

Fonte: elaborada pelos autores.

No trabalho de Silva Jr., Romano Jr. e Correia (2010), os autores inferem que os parâmetros de Densidade Proposicional (DP) e Conceitos Iniciais e Finais Múltiplos relativos (CIM e CFM) são bons indicadores do nível de proficiência dos alunos na elaboração dos MCs. Desse modo, o objetivo do presente trabalho foi avaliar e monitorar a proficiência dos alunos na técnica de mapeamento conceitual a partir destes três parâmetros estruturais da rede proposicional. A hipótese de trabalho considera que a estrutura do MC revela a evolução dos alunos durante o processo de treinamento, permitindo monitorar a proficiência ao longo de uma disciplina sobre Ciências. 


\section{Procedimentos de Pesquisa}

\section{Coleta de Dados}

Os dados foram coletados nos primeiros semestres letivos de 2009, 2010 e 2011, durante a disciplina Ciências da Natureza $(\mathrm{CN})$, oferecida a todos os alunos ingressantes na Escola de Artes, Ciências e Humanidades (EACH) da Universidade de São Paulo (USP). Com o objetivo de aumentar a alfabetização científica, a disciplina oferece uma visão abrangente sobre o impacto causado pelo desenvolvimento científico e tecnológico em nossa sociedade (ARAÚJO; FORMENTON, 2012; AULER, 1998; BYBEE; FUCHS, 2006; CORREIA et al., 2010; DEHAAN, 2005; DONNELLY, 2004; HOLBROOK; RANNIKMAE, 2007; LINSINGEN, 2007; SANTOS, 2007).

Os MCs coletados para análise $(\mathrm{n}=434)$ foram produzidos pelos alunos durante as $1^{\mathrm{a}}$ (MC1), $5^{\mathrm{a}}$ (MC5) e 15 (MC15) aulas da disciplina. As atividades da disciplina CN (Figura 2) contemplam a produção do MC1 colaborativo em trios sobre um assunto familiar aos alunos ingressantes no ensino superior (a dificuldade de ingressar na USP). Em seguida, eles passam por um período de treinamento de quatro aulas na técnica de mapeamento conceitual, abordando aspectos descritos e discutidos com detalhes na literatura (AGUIAR; CICUTO; CORREIA, 2014; AGUIAR; CORREIA, 2013). Os MC5 e MC15 foram elaborados individualmente em dois momentos formais de avaliação da disciplina envolvendo os temas Universo e Bioética, respectivamente. Os alunos foram incentivados a produzir MCs tanto de modo manuscrito (em papel) como utilizando o programa computacional gratuito CmapTools ${ }^{3}$ durante todas as 15 aulas da disciplina, estabelecendo, assim, um período de quatro meses de utilização sistemática da técnica.

Figura 2. Momentos de coleta de dados (434 MCs) durante o andamento da disciplina CN

2009-2010-2011 Ciências da Natureza (EACH/USP)

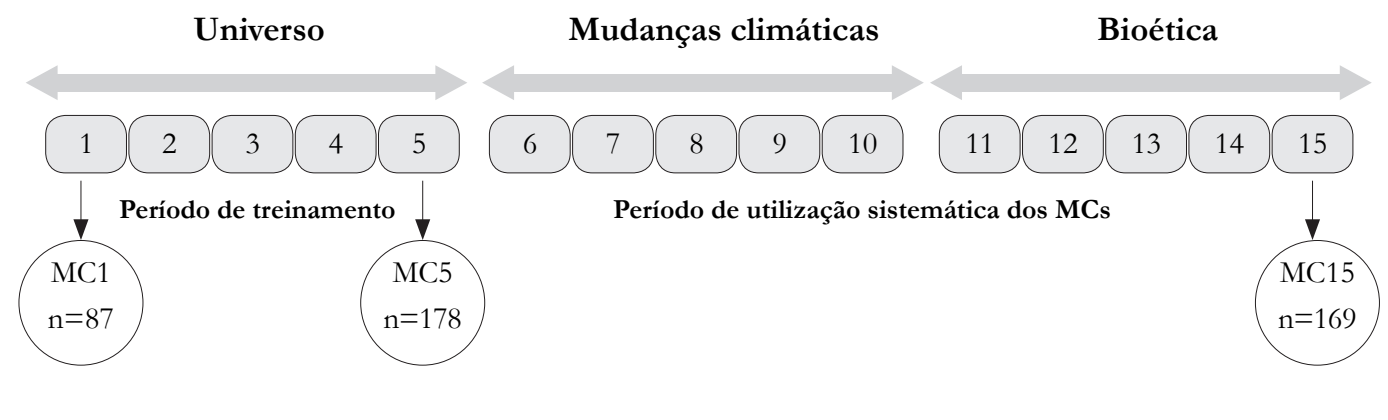

Fonte: elaborada pelos autores.

\footnotetext{
${ }^{3}$ Florida Institute for Human \& Machine Cognition (IHMC), Pensacola, EUA.
} 


\section{Análise dos Dados}

Primeiramente, foi contabilizada a quantidade de proposições, conceitos totais, iniciais múltiplos, finais múltiplos para cada um dos $434 \mathrm{MCs}$ produzidos durante a disciplina. Em seguida, os valores de DP, CIM e CFM foram determinados, conforme descrito na Tabela 1. Para avaliar a correlação entre os parâmetros estruturais, a soma CIM + CFM em função da DP foi representada em um gráfico de dispersão e a correlação linear de Person (r) foi calculada.

A partir das três variáveis da AE, realizou-se uma Análise Hierárquica de Agrupamentos (AHA), que consiste em uma análise multivariada de caráter exploratório (FIELD, 2009) cujo principal propósito é reunir objetos (nesse caso, os MCs) por sua similaridade em função das variáveis analisadas (nesse caso, os parâmetros da AE). Para estabelecer os cálculos necessários à análise foram considerados a distância Euclidiana (definida como a raiz quadrada do somatório das distâncias entre dois pontos $x$ e $y$ no plano cartesiano de $n$-dimensões) e o método Ward/ Incremental de iteração. Todas as análises foram feitas com auxílio do programa computacional IBM SPSS Statistics versão 22.0 (IBM Corporation, Armonk, NY, EUA).

As médias e desvios-padrão dos parâmetros da AE foram utilizados para caracterizar o padrão estrutural dos MCs em cada grupo da AHA. Para observar como os MCs produzidos ao longo da disciplina têm suas características estruturais alteradas, determinaram-se as frequências em porcentagem dos MCs 1, 5 e 15 presentes em cada um dos grupos, representadas através de um gráfico de barras.

\section{Resultados e Discussões}

\section{Correlação entre os parâmetros estruturais dos MCs}

O somatório dos conceitos múltiplos em função da densidade proposicional gerou o gráfico de dispersão apresentado na Figura 3, onde os pontos representam cada um dos 434 $\mathrm{MCs}$ produzidos durante a disciplina $\mathrm{CN}$.

A Figura 3 mostra que a quantidade de conceitos múltiplos (CIM + CFM) está relacionada a DP, apresentando uma forte correlação linear de Pearson $r=0,82$. Essa correlação pode ser explicada pelo efeito da restrição do número de conceitos do MC. Limitar a quantidade de conceitos totais para elaboração do MC exige que o aluno transforme conceitos a piori iniciais e/ou finais em conceitos múltiplos para gerar mais proposições. Isso implica no aumento do número de proposições sem aumentar o número de conceitos totais do MC. Como consequência, aumenta-se proporcionalmente o número de CIM e CFM, como mostra o gráfico da Figura 3. Essa discussão ressalta a importância de se comparar MCs elaborados sob as mesmas condições conforme já descrito na literatura por Cañas, Novak e Reiska (2015). Assim, cabe destacar que os MCs elaborados durante os momentos de coleta apresentavam uma quantidade total de 9 conceitos, arbitrariamente definida pelo professor da disciplina (P. R. M. C.) que considerou (i) o tempo disponível para a elaboração do MC (60 minutos) e (ii) o interesse em avaliar o poder de síntese dos alunos nas provas da disciplina. 
Correia, P. R. M.; Aguiar, J. G.

Figura 3. Correlação entre parâmetros da $\mathrm{AE}$ que estabelece um contínuo entre MCs produzidos por alunos ao longo da disciplina Ciências da Natureza de 2009 a 2011 (correlação de Pearson, r = 0,82)

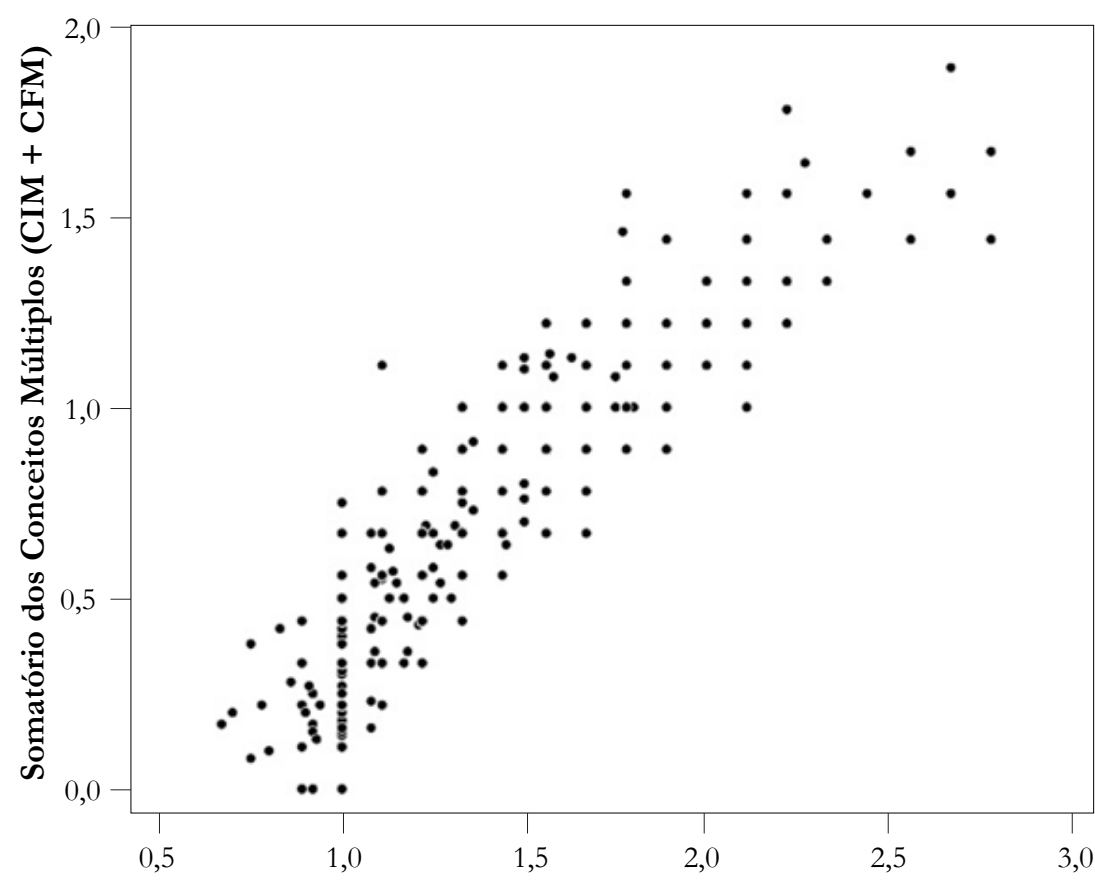

Densidade Proposicional (DP)

Fonte: elaborada pelos autores.

\section{Padrões estruturais dos MCs}

A aplicação da AHA para reunir os MCs em função dos seus parâmetros estruturais gerou um dendrograma que favorece a formação de quatro agrupamentos naturais (I-IV) com $71 \%$ de similaridade. Os valores médios com menores desvios-padrão indicam maior homogeneidade entre os MCs dentro de cada grupo. O número total de MCs, nos grupos de I a IV é 165, 174, 42 e 53, respectivamente. A análise de variância (ANOVA) indica que os grupos formados são estatisticamente diferentes tanto para os valores de DP, $F(431,3)=918,3, p=$ 0,00, como para as quantidades de CIM e CFM, $F(431,3)=535,6, p=0,00$ e $F(431,3)=457,06$, $p=0,00$, respectivamente. A caracterização e diferenciação dos grupos podem ser observadas no gráfico da Figura 4. 
Figura 4. Caracterização dos agrupamentos I-IV em função da média e desvios-padrão dos parâmetros estruturais dos MCs contidos em cada grupo

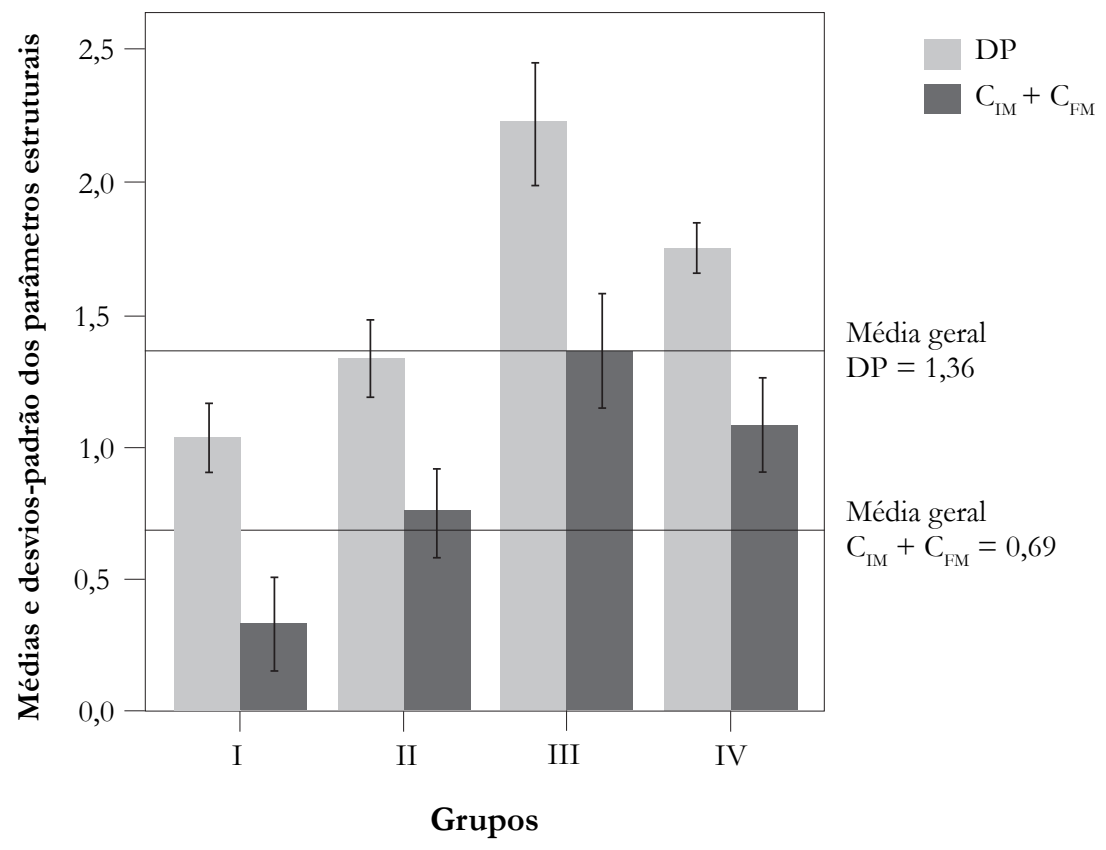

Fonte: elaborada pelos autores.

Cada agrupamento (I-IV) apresenta MCs com características estruturais nitidamente distintas. O grupo I apresenta a média dos parâmetros estruturais DP, CIM + CFM muito abaixo da média geral, indicando que esses MCs são pouco densos e articulados (38\% do total). Os MCs desse grupo apresentam uma proposição para cada conceito, sendo que dos nove conceitos, dois ou três são múltiplos. Já os MCs do grupo II (40\%) apresentam características em torno da média geral confirmando uma densidade ligeiramente maior que a do grupo I. Para cada três conceitos há aproximadamente quatro proposições sendo formadas e dos nove conceitos, ao menos, seis deles são classificados como múltiplos (finais ou iniciais). Os grupos III e IV apresentam MCs com parâmetros estruturais muito acima da média geral. O grupo III é formado por $12 \%$ de MCs extremamente densos e com conceitos exclusivamente múltiplos. Nesse caso, os MCs apresentam em média duas proposições para cada conceito do MC, sendo que, no mínimo, sete conceitos são ao mesmo tempo múltiplos iniciais e finais. Por fim, os MCs do grupo IV (10\%) são um pouco menos densos, configurando-se em uma estrutura que tem em média quinze proposições e nove conceitos múltiplos (iniciais ou finais). 


\section{Modificação dos padrões estruturais dos MCs ao longo da disciplina}

Para entender como o padrão estrutural dos MCs sofre alterações ao longo da disciplina e, consequentemente, compreender de que modo as atividades promovidas em sala de aula contribuíram para a aquisição de habilidades na técnica de mapeamento conceitual, a Figura 5 apresenta um gráfico de barras que indica a porcentagem relativa de cada grupo de MC nos três momentos analisados (1, 5 e 15) em relação ao total de MCs produzidos naquela aula em cada grupo formado pela AHA.

Figura 5. Porcentagem relativa de MCs produzidos nas aulas 1, 5 e 15 em cada grupo (I-IV)

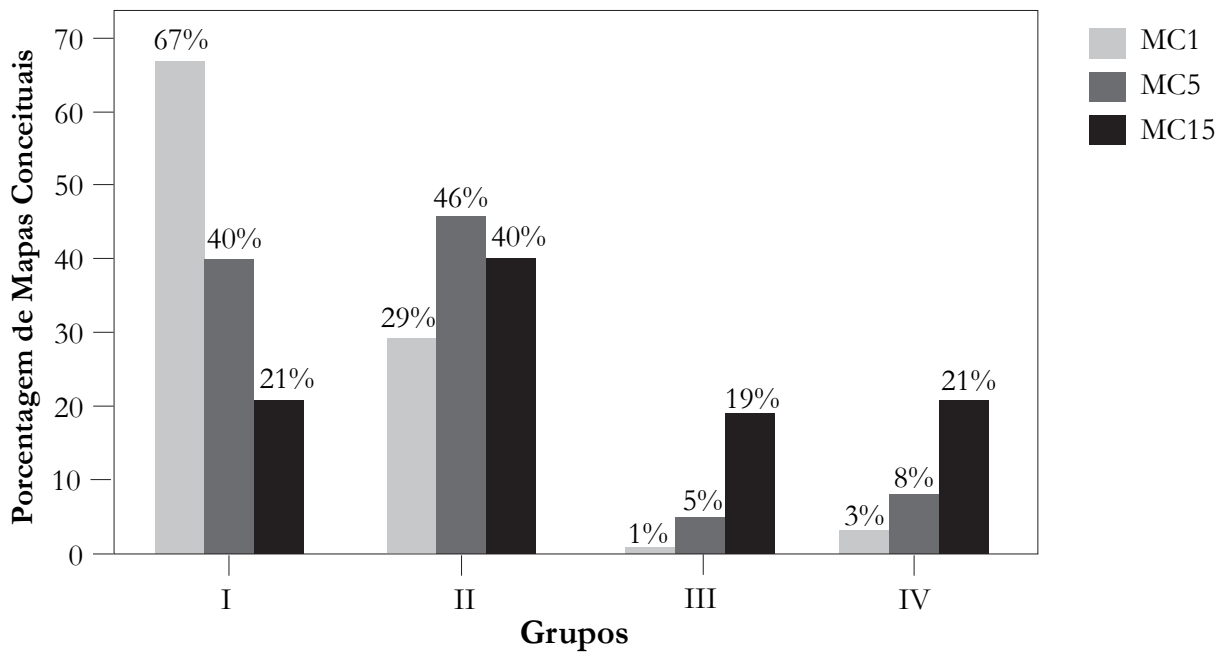

Fonte: elaborada pelos autores.

De acordo com os dados apresentados na Figura 5, os MCs produzidos na primeira aula da disciplina (MC1) estão predominantemente no grupo I (67\%). Esses MCs, elaborados no primeiro contato com a técnica, apresentam quantidade reduzida de proposições, que formam uma estrutura parecida com um texto. As quatro aulas seguintes da disciplina $\mathrm{CN}$ são destinadas às leituras e discussões científicas acerca do tema Universo, seguida de uma avaliação formal e individual na 5 aula da disciplina (MC5). Concomitantemente, atividades de treinamento são propostas como forma de garantir que os alunos compreendam os parâmetros de referência para elaborar bons MCs (AGUIAR; CORREIA, 2013). Conforme o gráfico da Figura 5, os MC5 estão distribuídos quase exclusivamente entre os grupos I (40\%) e II (46\%). A diminuição na proporção de MCs no grupo I e o aumento no grupo II, quando comparados ao MC1, demonstram que o período de treinamento oferece aos alunos meios de modificar a estrutura dos seus MCs, agora, com mais proposições para aumentar a articulação entre os conceitos. 
Nas 10 aulas seguintes da disciplina o professor propõe diversas tarefas de elaboração de MCs individuais e colaborativos, a fim de que os alunos se preparem para discutir desdobramentos sociais, ambientais, políticos e econômicos referentes aos temas Mudanças Climáticas e Bioética. Na última aula da disciplina o aluno realiza a terceira avaliação formal e individual sobre o tema Bioética (MC15). A Figura 5 indica que esses MCs estão distribuídos entre os quatro grupos, sendo com maior predominância no grupo II (40\%) e igualmente entre os grupos I e IV (21\%). A utilização sistemática do mapeamento conceitual em sala de aula tornou possível a elaboração de MCs com uma grande quantidade de proposições feitas a partir de conceitos amplamente articulados (grupo IV), o que pouco ocorreu no MC1 (apenas 3\%).

Se observarmos apenas os MCs com características estruturais muito acima da média geral (grupos III e IV) é possível perceber que somam apenas 4\% de MC1, progredindo para $13 \%$ de MC5, e aumentando para 40\% de MC15. Esses dados confirmam que as tarefas de treinamento ao longo da disciplina permitem aos alunos elaborar MCs cada vez mais densos e articulados. Na prática, pode-se dizer que os alunos se tornam capazes de lidar com a tarefa de elaboração dos MCs, ao mesmo tempo em que expressam melhor seus conhecimentos no tema abordado.

\section{Mapas conceituais ilustrativos de Ciências - casos mais frequentes}

Para ilustrar a mudança na estrutura ao longo da disciplina, um MC1 do grupo I (Figura 6a), um MC5 do grupo II (Figura 6b) e um MC15 característico do grupo IV (Figura 6c) são mostrados a seguir. Os termos de ligação foram suprimidos uma vez que a estrutura da rede proposicional é o que interessa discutir nesse momento.

Ao observarmos os MCs dos alunos (Figura 6) é possível perceber visualmente que as estruturas das redes proposicionais são diferentes. Todos possuem a mesma quantidade de conceitos totais (nove), porém, o aluno que elaborou o MC1 (Figura 6a) utilizou apenas oito proposições para responder à pergunta focal $(\mathrm{DP}=0,89)$, enquanto os alunos que elaboraram os MC5 (Figura 6b) e MC15 (Figura 6c) utilizaram 12 (DP = 1,34) e 17 (DP = 1,89) proposições, respectivamente. $\mathrm{O}$ aumento da DP ao longo da disciplina poderia ser explicado pelo maior entendimento no conteúdo. Porém, os assuntos abordados na $1^{a}$ aula (MC1) e nas duas provas (MC5 e MC15) são completamente diferentes entre si. Esse aumento de DP deve-se a um maior entendimento da técnica e da capacidade do aluno em estabelecer mais proposições para explicitar seu conhecimento no tema.

A integração da rede é dada pela maior conexão entre conceitos. No caso do MC1 (Figura 6a), o encadeamento de conceitos é semelhante a um texto, onde sete dos nove conceitos presentes no MC estão ligados diretamente a apenas outro conceito subordinado a ele. Com isso os parâmetros relativos aos conceitos múltiplos são muito baixos (CIM $+\mathrm{CFM}=0,22)$, o que caracteriza os MCs do grupo I. Apesar de o tema mapeado ser extremamente familiar a esse aluno, o MC se revela pouco denso e articulado. Isso pode ser explicado, pois, nessa tarefa o aluno tem que lidar cognitivamente com duas demandas relativas (1) ao conteúdo (recuperar informações relativas ao seu conhecimento) e (2) à forma (se expressar utilizando um organizador gráfico desconhecido).

No caso do MC5 (Figura 6b), um conceito de base (mais tecnologia) expande a rede proposicional através da sua conexão com quatro conceitos diretamente subordinados a ele 
(Big Bang, aglomerado de galáxias, observações e telescópio). A diferença do MC5 para o MC1 (Figura 6a) é a presença de alguns conceitos que agora podem servir de conexão entre diferentes níveis de conhecimento. Por exemplo, os conceitos Big Bang e ovo cósmico remetem à criação do Universo, enquanto os conceitos Galileu e telescópio remetem aos avanços científico-tecnológicos que permitiram maior observação do Universo. No caso, o aluno não explicitou qualquer ligação direta a esses conceitos, porém, utilizou um conceito em comum (evidências) para isso. Essa manipulação faz com que a quantidade de proposições (DP = 1,34) e conceitos múltiplos $(\mathrm{CIM}+\mathrm{CFM}=0,66)$ aumente mais em relação a um $\mathrm{MC}$ essencialmente linear como é o caso do MC1.

A organização conceitual hierárquica é considerada um ponto crítico para elaboração de bons MCs. No caso do MC5, a capacidade do aluno em escolher conceitos relacionados ao tema e dispô-los espacialmente no MC para formular proposições já demonstra familiaridade com a técnica de mapeamento conceitual. Durante a sua elaboração, o aluno não conseguiu usufruir do seu poder de escolha dos conceitos nem da proximidade espacial provida pelo organizador gráfico. Como consequência, a rede proposicional se apresenta ainda pouco densa e pouco articulada entre si, característica central dos MCs do grupo II.

Figura 6. MCs com nove conceitos e pergunta focal predefinida produzidos por alunos durante a disciplina $\mathrm{CN}$

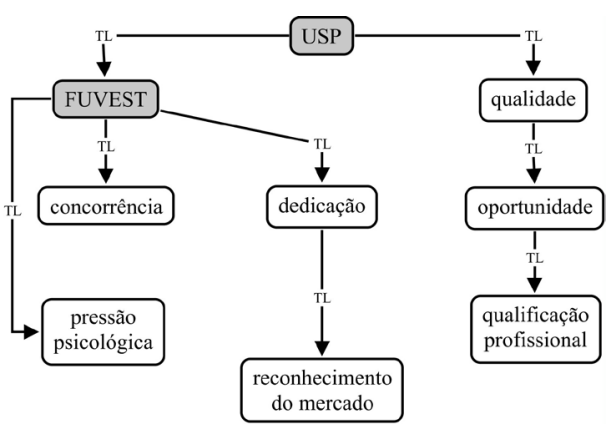

(a)
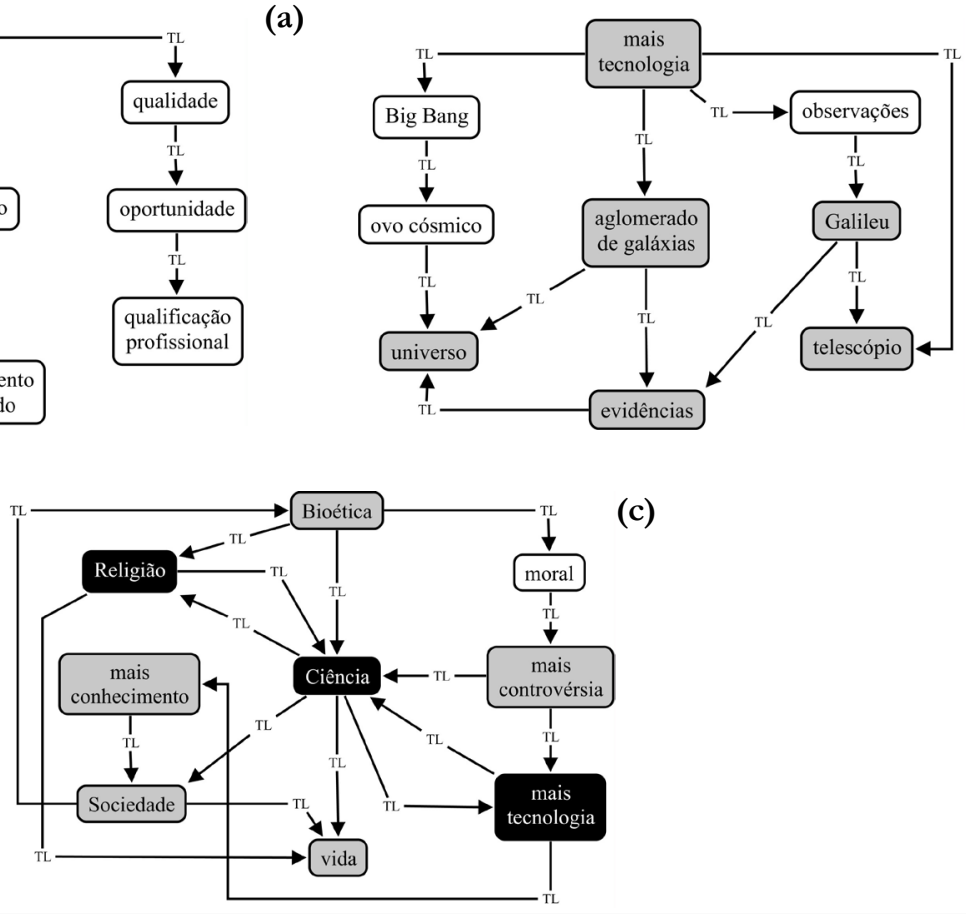

(a) MC1 do grupo I (tema: a dificuldade de ingresso na USP); (b) MC5 do grupo II (tema: Universo); (c) MC15 do grupo IV (tema: Bioética). Legenda: fundo branco - conceito ordinário; fundo cinza - conceito múltiplo inicial ou final; fundo preto - conceito múltiplo inicial e final; TL - termo de ligação.

Fonte: elaborada pelos autores a partir dos originais entregues pelos estudantes. 
Por fim, o MC15 (Figura 6c) pode ser classificado propriamente como uma rede de conceitos onde sua hierarquia é menos óbvia, uma vez que oito dos nove conceitos estão ligados a outros dois ou mais conceitos. Isso significa que, para aumentar a integração da rede de proposições sem alterar o número de conceitos, o aluno precisou elaborar muitas proposições utilizando os mesmos conceitos, atribuindo a estes a característica de serem múltiplos (iniciais ou finais). Como consequência, os parâmetros da $\mathrm{AE}$ se tornaram relativamente altos (DP = 1,89; CIM + CFM = 1,13), característica essencial dos MCs do grupo IV. O aluno que elaborou o MC15 foi capaz de escolher conceitos-chave sobre o tema (p. ex., Bioética, Ciência, moral, Sociedade), dispô-los espacialmente da melhor forma possível, perceber e realizar as conexões necessárias entre diferentes níveis de conhecimento (p. ex., Religião e moral interligadas à Sociedade e vida por meio da Ciência e Bioética). Essa manipulação do organizador gráfico só é possível quando o aluno é proficiente na técnica de mapeamento conceitual e conhece razoavelmente o tema mapeado.

Vale destacar que o senso comum que reveste as discussões sobre temas controversos como a criação, expansão e estudo do Universo (tema do MC5) ou de como a ética, moral e religião influenciam o estudo sobre a clonagem e terapia gênica (tema do MC15), poderiam dar base para um aluno proficiente na técnica elaborar um MC ingênuo. Porém, os MCs vistos nas Figuras $6 \mathrm{~b}$ e $6 \mathrm{c}$ não poderiam ter sido feitos por especialistas em Universo/Bioética que desconhecem os princípios da técnica de mapeamento. Em outras palavras, é necessário o treinamento na técnica até mesmo para que um especialista (professor) possa expressar conhecimento organizado na forma de MCs.

\section{Mapas conceituais ilustrativos de Ciências - casos especiais}

Além dos MCs apresentados na seção anterior, oferecemos ainda dois casos particulares: um MC15 do grupo I (Figura7a) e um MC5 do grupo III (Figura 7b). Nessa seção os termos de ligação são oferecidos para permitir uma segunda dimensão de análise desses MCs, a análise de conteúdo ou correção conceitual.

$\mathrm{Na}$ Figura $7 \mathrm{a}$ é possível visualizar um MC15 pouco denso $(\mathrm{DP}=1,00)$ e com poucas articulações entre os conceitos (CIM $+\mathrm{CFM}=0,33)$, ilustrando um caso particular de um aluno que passou pelo período de treinamento e pelas 14 aulas da disciplina com tarefas para elaboração de MCs acompanhada de discussões sobre os conteúdos científicos relativos ao Universo, Mudanças Climáticas e Bioética. Analisando esse MC, percebe-se que há presença de clareza semântica nas proposições (p. ex. inovações - geram $\rightarrow$ mais controvérsia). Mesmo havendo pertinência dos conceitos escolhidos (p. ex. clonagem, transgenia e Sociedade), as proposições guardam certa ingenuidade (p. ex. genética - é uma área da $\rightarrow$ Ciência ou Bioética - considera $\mathrm{o} \rightarrow$ ético), nos levando a inferir que esse aluno sabe expressar seu conhecimento na forma de $\mathrm{MC}$, porém, não compreendeu com profundidade as discussões relativas ao tema.

No caso da Figura 7b é apresentado um MC5 extremamente denso (DP $=2,11)$ e integrado $(\mathrm{CIM}+\mathrm{CFM}=1,56)$, ilustrando outro caso especial de um aluno que apresenta total domínio sobre a técnica de mapeamento conceitual após o período de treinamento de 4 aulas concomitante às discussões em sala sobre o tema Universo. Analisando esse MC, percebe-se que as proposições têm clareza semântica (p. ex. Sociedade - gera $\rightarrow$ novos cientistas), os conceitos escolhidos são pertinentes (p. ex. Ciência, Sociedade, pesquisa, Universo) e não há proposições 
ingênuas no MC. É possível que esse aluno já possuísse conhecimento prévio sobre o tema e, com isso, os recursos cognitivos disponíveis na sua memória de trabalho foram alocados para lidar com a tarefa de elaboração do MC (CORREIA; AGUIAR, 2014).

Garantir a proficiência do aluno na construção de MC abre espaço para que o professor identifique proposições que revelam limitações e/ou erros conceituais (NOVAK, 2002). Por exemplo, na proposição: "dogmas e religião - se opunham a existência do $\rightarrow$ Universo", o professor pode mencionar ao aluno que a Igreja e seus dogmas não se opõem à existência do Universo. A explicação religiosa sobre a origem e a evolução do Universo difere da científica, mas nenhuma delas nega tal existência. De forma análoga, a proposição "pesquisa - ajuda a provar o $\rightarrow$ Universo", abre espaço para discutir a concepção do aluno sobre o significado de que "provar" leva eventualmente a uma verdade absoluta, o que não condiz com a compreensão adequada do conhecimento produzido cientificamente, que deve ser aceito e validado dentro de um contexto histórico.

Figura 7. MCs com nove conceitos, pergunta focal e estrutura pré-definida produzidos por alunos durante $\mathrm{CN}$

(a)

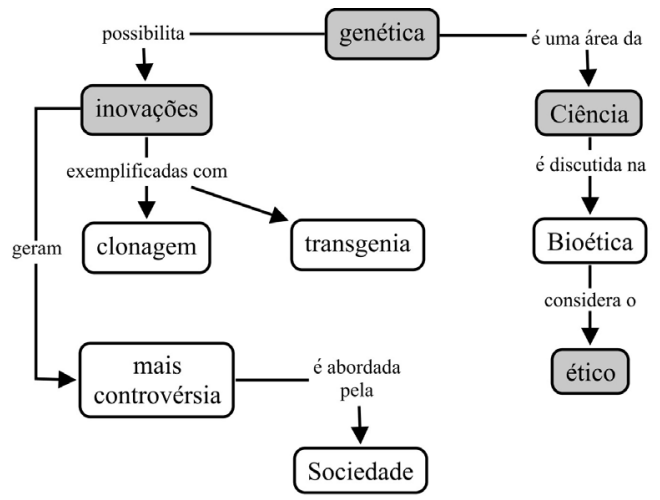

(b)

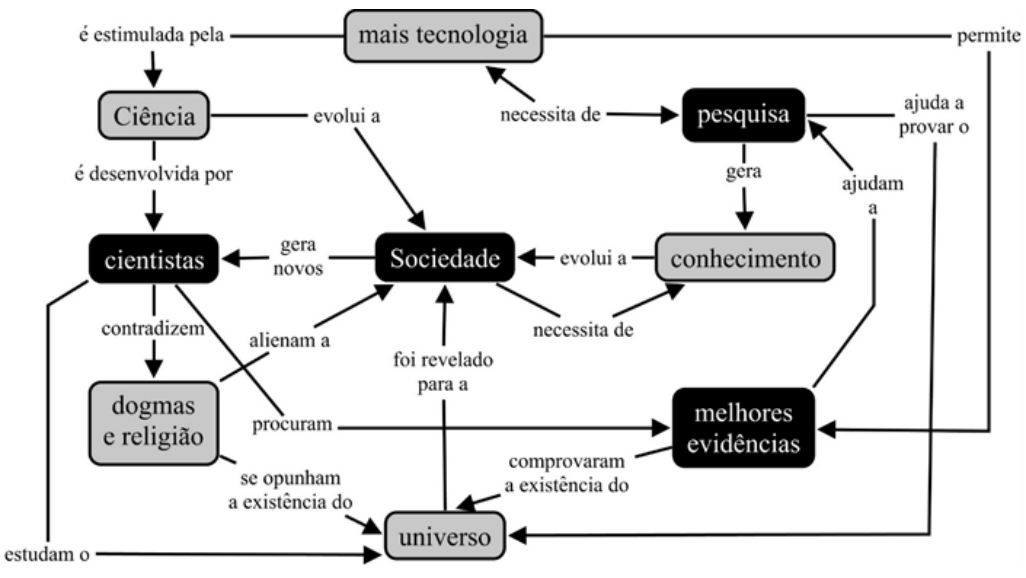

(a) MC15 do grupo I (tema: Bioética); (b) MC5 do grupo III (tema: Universo). Legenda de cores: fundo branco conceito ordinário; fundo cinza - conceito múltiplo inicial ou final; fundo preto - conceito múltiplo inicial e final. Fonte: elaborada pelos autores partir dos transcritos dos originais entregues pelos estudantes. 


\section{Limitações e perspectivas futuras da análise estrutural}

Independentemente do tema mapeado, a presença de proficiência não implica necessariamente em maior ou menor compreensão do tema. Entende-se que o primeiro passo da análise de um MC produzido por um aluno seja observar os seus parâmetros estruturais, ou seja, determinar a densidade proposicional e a quantidade de conceitos múltiplos. Garantida a proficiência, os passos seguintes devem ser dados no sentido de (1) contemplar a leitura de cada proposição e o julgamento quanto a sua clareza semântica, pertinência e validade conceitual frente aos conteúdos tratados durante a disciplina; (2) observar se a rede proposicional como um todo é suficientemente capaz de responder à pergunta focal estabelecida pelo professor; (3) julgar se a hierarquia conceitual foi estabelecida pelo aluno e, em caso positivo, se a mesma auxiliou na organização e articulação do conteúdo de forma eficiente e correta.

Como perspectiva futura, vislumbra-se a possibilidade de elaboração de um modelo algorítmico capaz de determinar o nível de proficiência do mapeador a partir da combinação univariada ou multivariada dos parâmetros estruturais do MC. Com esse modelo esperamos atingir maior autonomia, otimização e agilidade nessa análise estrutural prévia, auxiliando tanto no ensino presencial como a distância no que diz respeito à verificação da proficiência dos alunos na técnica de maepamento conceitual.

\section{Conclusões}

Expressar o conhecimento científico na forma de MC requer claro entendimento da técnica de mapeamento conceitual. Tal entendimento torna possível a construção de MCs com alta densidade de proposições (DP) e conceitos articulados entre si (CIM + CFM) para representar o conhecimento e suas relações conceituais.

A partir dos resultados apresentados, foi possível concluir que esses três parâmetros estruturais (DP, CIM e CFM) foram capazes de descrever o aumento progressivo de capacitação na técnica ao longo de uma disciplina sobre as Ciências Naturais. O período de treinamento de quatro aulas e a utilização sistemática da técnica por mais 11 aulas foram fatores críticos para garantir os bons resultados apresentados.

É importante realçar que a $\mathrm{AE}$ não revela o grau de entendimento conceitual que o aluno tem a respeito do tema e, por isso, a leitura e interpretação das proposições do MC complementam a análise descrita nesse trabalho. Entretanto, entendemos que a capacitação na técnica de mapeamento conceitual permite revelar de modo mais fidedigno as representações mentais dos alunos sobre determinado conteúdo e, portanto, uma análise de pertinência, validade e correção conceitual das proposições do MC tende a ser favorecida se garantido o prévio treinamento na técnica. 


\section{Agradecimentos}

Os autores agradecem às agências de fomento à pesquisa que financiam os trabalhos desenvolvidos pelo nosso grupo de pesquisa: ao Conselho Nacional de Desenvolvimento Científico e Tecnológico (CNPq-486194/2011-6) e à Fundação de Amparo à Pesquisa do Estado de São Paulo (FAPESP-2012/22693-5).

A autora Joana Guilares de Aguiar agradece à Coordenação de Aperfeiçoamento de Pessoal de Nível Superior (CAPES) pela bolsa de doutorado.

\section{Referências}

AGUIAR, J. G.; CICUTO, C. A. T.; CORREIA, P. R. M. How can we prepare effective concept maps?: training procedures and assessment tools to evaluate mappers' proficiency. Journal of Science Education, Bogota, v. 15, n. 1, p. 14-19, 2014. Disponível em: <http:// www.accefyn.org.co/rec/portal/>. Acesso em: 3 jan. 2017.

AGUIAR, J. G.; CORREIA, P. R. M. Como fazer bons mapas conceituais?: estabelecendo parâmetros de referências e propondo atividades de treinamento. Revista Brasileira de Pesquisa em Educação em Ciências, Belo Horizonte, v. 13, n. 2, p. 141-157, 2013. Disponível em: < https://seer.ufmg.br/index.php/rbpec/article/view/2469>. Acesso em: 3 jan. 2017.

ARAÚJO, M. S. T.; FORMENTON, R. As fontes de energia automotiva abordadas sob o enfoque CTS no ensino médio profissionalizante. Alexandria, Florianópolis, v. 5, n. 1, p. 33-61, 2012. Disponível em: < https://periodicos.ufsc.br/index.php/alexandria/article/ view/37696>. Acesso em: 3 jan. 2017.

AULER, D. Movimento ciência-tecnologia-sociedade (CTS): modalidades, problemas e perspectivas em sua implementação no ensino de física. In: ENCONTRO DE PESQUISA EM ENSINO DE FÍSICA, 6, 1998, Florianópolis. Resumos... Florianópolis: SBF, 1998.

AUSUBEL, D. P. The acquisition and retention of knowledge: a cognitive view. Dordrechet: Kluwer, 2000.

BYBEE, R. W.; FUCHS, B. Preparing the $21^{\text {st }}$ century workforce: a new reform in science and technology education. Journal of Research in Science Teaching, Hoboken, v. 43, n. 4, p. 349-352, 2006. Disponível em: <https://doi.org/10.1002/tea.20147>. Acesso em: 3 jan. 2017.

CAÑAS, A. J.; BUNCH, L.; REISKA, P. CmapAnalysis: an extensible concept map analysis tool. In: CAÑAS, A. J.; NOVAK, J. D.; SANCHEZ, J. (Ed.). Proceedings of the fourth International Conference on Concept Mapping. Viña del Mar: Universidad de Santiago, 2010. v. 1, p. 73-83. Disponível em: <http://cmc.ihmc.us/cmc2010papers/cmc2010-a13. pdf>. Acesso em: 3 jan. 2017. 
Avaliação da proficiência em mapeamento conceitual ...

CAÑAS, A. J.; NOVAK, J. D. Re-examining the foundations for effective use of concept maps. In: __ (Ed.). Proceedings of the second International Conference on Concept Mapping. San Jose: Universidad de Costa Rica, 2006. v. 1, p. 494-502. Disponível em: <http://cmc.ihmc.us/cmc2006Papers/cmc2006-p247.pdf>. Acesso em: 3 jan. 2017.

CAÑAS, A. J.; NOVAK, J. D.; REISKA, P. How good is my concept map?: am I a good Cmapper? Knowledge Management \& E-Learning, Hong Kong, v. 7, n. 1, p. 6-19, 2015. Disponível em: <http://www.kmel-journal.org/ojs/index.php/online-publication/article/ view/407/244>. Acesso em: 3 jan. 2017.

CAÑAS, A. J. et al. Confiabilidad de una taxonomía topológica para mapas conceptuales. In: CAÑAS, A. J.; NOVAK, J. D. (Ed.). Proceedings of the second International Conference on Concept Mapping. San Jose: Universidad de Costa Rica, 2006. v. 1, p. 153-161. Disponível em: <http://cmc.ihmc.us/cmc2006Papers/cmc2006-p233.pdf>. Acesso em: 3 jan. 2017.

CONRADTY, C.; BOGNER, F. X. Implementation of concept mapping to novices: reasons for errors, a matter of technique or content? Educational Studies, Philadelphia, v. 36, n. 1, p. 47-58, 2010. Disponível em: < https://doi.org/10.1080/03055690903148605>. Acesso em: 3 jan. 2017.

CORREIA, P. R M.; AGUIAR, J. G. Concept mapping informed by cognitive load theory: implications for tasks involving learner-generated Cmaps. In: CORREIA, P. R. M. et al. (Ed.). Proceedins of the sixth International Conference on Concept Mapping. Santos: USP, 2014. v. 1, p. 150-157. Disponível em: <http://cmc.ihmc.us/cmc2014papers/cmc2014-p150. pdf>. Acesso em: 3 jan. 2017.

CORREIA, P. R M.; INFANTE-MALACHIAS, M. E.; GODOY, C. E. C. From theory to practice: the foundations for training students to make collaborative concept maps. In: CAÑAS, A. J. et al. (Ed.). Proceedings of the third International Conference on Concept Mapping. Tallinn: OÜ Vali Press, 2008. v. 2, p. 414-421. Disponível em: <http:// cmc.ihmc.us/cmc2008papers/cmc2008-p146.pdf>. Acesso em: 3 jan. 2017.

CORREIA, P. R M. et al. The importance of scientific literacy in fostering education for sustainability: theoretical considerations and preliminary findings from a Brazilian experience. Journal of Cleaner Production, Amsterdam, v. 18, n. 7, p. 678-685, 2010. Disponível em: <https://doi.org/10.1016/j.jclepro.2009.09.011>. Acesso em: 3 jan. 2017.

CORREIA, P. R M. et al. Por que vale a pena usar mapas conceituais no ensino superior? Revista de Graduação USP, São Paulo, v. 1, n. 1, p. 41-52, 2016. Disponível em: <http:// gradmais.usp.br/wp-content/uploads/2016/07/05_Correia.pdf>. Acesso em: 3 jan. 2017.

DAVIES, M. Concept mapping, mind mapping and argument mapping: what are the differences and do they matter? Higher Education, Dordrecht, v. 62, n. 3, p. 279-301, 2011. Disponível em: <https://doi.org/10.1007/s10734-010-9387-6>. Acesso em: 3 jan. 2017.

DEHAAN, R. L. The impending revolution in undergraduate science education. Journal of Science Education and Technology, Dordrecht, v. 14, n. 2, p. 253-269, 2005. Disponível em: <https://doi.org/10.1007/s10956-005-4425-3>. Acesso em: 3 jan. 2017. 
Correia, P. R. M.; Aguiar, J. G.

DONNELLY, J. F. Humanizing science education. Science Education, Hoboken, v. 88, n. 5, p. 762-784, 2004. Disponível em: <https://doi.org/ 10.1002/sce.20004>. Acesso em: 3 jan. 2017.

FIELD, A. Descobrindo a estatística usando o SPSS. 2. ed. Porto Alegre: Artmed, 2009.

FISCHER, F. et al. Fostering collaborative knowledge construction with visualization tools. Learning and Instruction, Kidlington, v. 12, n. 2, p. 213-232, 2002. Disponível em: <https://doi.org/10.1016/S0959-4752(01)00005-6>. Acesso em: 3 jan. 2017.

GERSTNER S.; BOGNER, F. Concept map structure, gender and teaching methods: an investigation of students' science learning. Educational Research, Abingdon, v. 51, n. 4, p. 425- 438, 2009. Disponível em: <https://doi.org/10.1080/00131880903354758>. Acesso em: 3 jan. 2017.

HAY, D.; KINCHIN, I; LYGO-BAKER, S. Making learning visible: the role of concept mapping in higher education. Studies in Higher Education, Abingdon, v. 33, n. 3, p. 295-311, 2008. Disponível em: <https://doi.org/10.1080/03075070802049251>. Acesso em: 3 jan. 2017.

HILBERT, T. S.; RENKL, A. Concept mapping as a follow-up strategy to learning from texts: what characterizes good and poor mappers? Instructional Science, Dordrecht, v. 36, n.1, p. 53-73, 2008. Disponível em: < https://doi.org/10.1007/s11251-007-9022-9>. Acesso em: 3 jan. 2017.

HOLBROOK, J.; RANNIKMAE, M. The nature of science education for enhancing scientific literacy. International Journal of Science Education, Abingdon, v. 29, n. 11, 1347-1362, 2007. Disponível em: <https://doi.org/10.1080/09500690601007549>. Acesso em: 3 jan. 2017.

KARPICKE, J. D.; BLUNT, J. R. Response to comment on "Retrieval practice produces more learning than elaborative studying with concept mapping". Science, Washington, v. 334, n. 6055, p. 453, 2011. Disponível em: <https://doi.org/10.1126/science.1204035>. Acesso em: 3 jan. 2017.

KINCHIN, I. M.; ALIAS, M. Exploiting variations in concept map morphology as a lessonplanning tool for trainee teachers in higher education. Journal of In-service Education, Abingdon, v. 31, n. 3, p. 569-92, 2005.

KINCHIN, I. M.; HAY, D. B.; ADAMS, A. How a qualitative approach to concept map analysis can be used to aid learning by illustrating patterns of conceptual development.

Educational Research, Abingdon, v. 42, n.1, p. 43-57, 2000. Disponível em: <https://doi. org/10.1080/001318800363908>. Acesso em: 3 jan. 2017.

LINSINGEN, I. Perspectiva educacional CTS: aspectos de um campo em consolidação na América Latina. Ciência \& Ensino, Piracicaba, v. 1, número especial, p. 1-16, 2007. Disponível em: <http://prc.ifsp.edu.br/ojs/index.php/cienciaeensino/article/ view/150/108>. Acesso em: 3 jan. 2017. 
Avaliação da proficiência em mapeamento conceitual ...

MOON, B. M. et al. Applied concept mapping: capturing, analyzing, and organizing knowledge. Boca Raton: CRC, 2011.

MOREIRA, M. A. Aprendizagem significativa: a teoria e textos complementares. São Paulo: Livraria da Física, 2011.

NESBIT, J. C.; ADESOPE, O. O. Learning with concept and knowledge maps: a metaanalysis. Review of Educational Research, Thousand Oaks, v. 76, n. 3, p. 413-448, 2006. Disponível em: <http://www.sfu.ca/ jcnesbit/articles/NesbitAdesope2006.pdf >. Acesso em: 3 jan. 2017.

NOVAK, J. D. Concept mapping: a useful tool for science education. Journal of Research in Science Teaching, Hoboken, v. 27, n. 10, p. 937-949, 1990. Disponível em: <https://doi. org//10.1002/tea.3660271003>. Acesso em: 3 jan. 2017.

. Learning, creating, and using knowledge: concept maps as facilitative tools in schools and corporations. New York: Routledge, 2010.

. Meaningful learning: the essential factor for conceptual change in limited or inappropriate propositional hierarchies leading to empowerment of learners. Science Education, Hoboken, v. 86, n.4, p. 548-571, 2002. Disponível em: < https://doi. org/10.1002/sce.10032>. Acesso em: 3 jan. 2017.

NOVAK, J. D.; MUSONDA, D. A twelve-year longitudinal study of science concept learning. American Educational Research Journal, Thousand Oaks, v. 28, n. 1, p. 117-153, 1991.

PAIVIO, A. Mental representations: a dual coding approach. Oxford: Oxford University Press, 1990.

RUIZ-PRIMO, M. A.; SHAVELSON, R. J. Problems and issues in the use of concept maps in science assessment. Journal of Research in Science Teaching, Hoboken, v. 33, n. 6, p. 569-600, 1996. Disponível em: < https://doi.org/10.1002/(SICI)10982736(199608)33:6<569::AID-TEA1>3.0.CO;2-M>. Acesso em: 3 jan. 2017.

SANTOS, W. L. P. Scientific literacy: a freirean perspective as a radical view of humanistic science education. Science Education, Hoboken, v. 93, n. 2, 361-382, 2007. Disponível em: <https://doi.org/10.1002/sce.20301>. Acesso em: 3 jan. 2017.

SILVA JR., S. N.; ROMANO JR., J. G.; CORREIA, P. R. M. Structural analysis of concept maps to evaluate the students' proficiency as mappers. In: CAÑS, A. J.; NOVAK, J. D.; SANCHEZ, J. (Ed.). Proceedings of the fourth International Conference on Concept Mapping. Viña del Mar: Universidad de Santiago, 2010. v. 1, p. 369-376. Disponível em: <http:/ /cmc.ihmc.us/cmc2010papers/cmc2010-b14.pdf>. Acesso em: 4 jan. 2017.

STARR, M. L.; KRAJCIK, J. S. Concept maps as a heuristic for science curriculum development: toward improvement in process and product. Journal of Research in Science Teaching, Hoboken, v. 27, n.10, p. 987-1000, 1990. Disponível em: <https://doi. org/10.1002/tea.3660271007>. Acesso em: 4 jan. 2017. 
Correia, P. R. M.; Aguiar, J. G.

SWELLER, J.; AYRES, P.; KALYUGA, S. Cognitive load theory. New York: Springer, 2011.

TORRES, P. L.; MARRIOTT, R. C. V. Handbook of research on collaborative learning using concept mapping. Hershey: IGI Global, 2010.

VEKIRI, I. What is the value of graphical displays in learning? Educational Psychology Review, New York, v. 14, n. 3, p. 261-312, 2002. Disponível em: < https://doi. org/10.1023/A:1016064429161>. Acesso em: 4 jan. 2017.

WANDERSEE, J. H. Concept mapping and the cartography of cognition. Journal of Research in Science Teaching, Hoboken, v. 27, n. 10, p. 923-936, 1990. Disponível em: <https://doi.org/10.1002/tea.3660271002>. Acesso em: 4 jan. 2017.

Artigo recebido em 14/07/2015. Aceito em 22/08/2016.

Endereço para contato: USP, Escola de Artes, Ciências e

Humanidades, Avenida Arlindo Bettio, 1000 Prédio I-1, Sala 320/D,

São Paulo, SP, CEP 03828-000, Brasil. 